\title{
La orientación profesional
}

\author{
SUS PROBLEMAS Y SUS MÉTODOS
}

Dor el Dr. Claparède.

AMOS a situar la Orientación Profesional en el conjunto de disciplinas que tienen por objeto el esludio cientifico del trabajo y de sus condiciones; es decir, en el conjunto de la psicologia aplicada al comercio, a la índustria y, de un modo general. a las profesiones.

El problema de la Orienlación Profesional nació de la necesidad de adaptar el trabajo al trabajador, y vice-versa.

Desde antiguo. los individuos poco eficientes eran excluidos por instinto de sus funciones. Pero nada se habia hecho, hasta hace poco por determinar cuál era el trabajo apropiado a cada individuo. Fué el filäntropo Parson quien, interesándose a principios del siglo XX por los muchachos vagabundos de New York. se dedicó a descubrir la situación que mejor convenia a cada uno de ellos, interrogándolos, de un modo sistemático, respecto de sus guslos, aptitudes, lecturas, etc.

En cuanto a la escuela. los educadores no habian pensado en preocuparse de la profesión que más tarde abrazarian sus alumnos. Educar era para ellos formar las cualidades generales e inculcar los conocimientos elementales que están sobre todas las especialidades.

Hoy se combate cada vez más esta manera de educar. La - escuela no eslá hecha para la escuela, dice M. Buisson. Debe - preparar hombres para la sociedad de mañana. Abandonar 
- bruscamente a sus jóvenes alumnos es una cruel irrisión, puesto

- que los arroja desarmados en plena batalla. Es desperdiciarlo - todo de un modo insensato. IQué locura hacer tanto por el - escolar y no preocuparse del aprendiz.... .

De esta nueva tendencia nace. pues. la necesidad de que el Maestro aprenda a descubrir las muestras de talento en germen. a despertar en el niño el propio conocimiento de sus capacidades especiales, imponiéndose el deber de ejercitarlas para su porvenir.

Tampoco la psicología aplicada, ni la psicología experimental han permanecido indiferentes ante el problema de las aptitudes. Esta última, sobre todo, se ha esforzado mediante la elaboración de pruebas especiales llamadas Tests mentales, por determinar, analizar las diversas particularidades psicológicas del individuo, buscando lo que le distingue, lo que le diferencia de los demás (psicologia individual).

Finalmente, la guerra última ha venido a acelerar este movimiento relativo al problema de las aptitudes.

Orientación y Selección:-Vamos a distinguir claramente entre estos dos problemas.

El primero se refiere a la elección de un individuo para una profesión.

El problema de la orientación se refiere a la elección de una profesión para un individuo.

Inoficioso sería entrar a delallar la importancia del último de estos problemas, ya que al emprender la tarea de dirigir a un adolescente, a un adulto sin trabajo o a un mutilado hacia la profesión que le ofrezca más probabilidades de éxito, no sólo va a conseguirse la felicidad de ese individuo, sino también el beneficio de los intereses de la colectividad.

El gran problema de la Orientación Profesional será, pues. el de determinar las aptitudes de un canditato.

Pero, de antemano, habrá que conocer cuáles son las aptifudes requeridas para las diversas profesiones, cuáles son sus exigencias, peligros, influencia sobre la salud, duración del aprendizaje, salario, etc. 
Se puede llegar a obtener este conocimiento, o sea, al análisis psicofisiológico de las profesiones:

1. Dor medio de las encuestas, conversaciones, cuestionarios, etc.

2. Por la observación simple, y

3. Por medio de la experimentación, o sea, de la determinación precisa de los lactores que entran en juego en el fenómeno que se estudia.

Ya analizadas en esta forma las profesiones, abria que entrar a clasificarlas.

Psicológicamente, podria clasificárselas (Piorkowski) en profesiones calificadas y no calificadas, según su ejercicio exija o no aptitudes especiales.

Las profesiones calificadas se subdividen en especializadas [ponen en acción ciertas formas de la atención y cierlas reacciones. siempre las mismas, (obreros de las fábricas. mineros, etc.)]: profesiones medias (exigen ya cierta suma de inteligencia y cierla combinación de aptitudes psiquicas: tipógrafos. lelefonistas, dactilógrafos, profesores de gimnasia, música y lenguas). y las profesiones superiores (exigen, ante todo. inteligencia creadora: ingenieros, arquitectos, médicos, etc.)

Una vez establecidas y convenienternente clasificadas las cualidades requeridas para las diversas profesiones, volvamos al problema que habiamos dejado pendiente, es decir, a determinar. a descubrir las diversas aplifudes de un individuo.

Podemos servirnos para ello de los mismos mélodos que usamos para el análisis de las profesiones: $10^{\circ}$ interrogarorio (del sujeto y del medio que lo rodea); $2 .^{\circ}$ observación; y $3 .^{\circ}$ la experimentación.

Sin desconocer, por cierlo. la dificultad de conocerse a si mismo, se pueden pedir al sujeto informaciones respecto de sus condiciones familiares, salud, fisiologia, hábitos, juegos, conocimientos, ambiciones, cualidades intelectuales y morales, vicios. defectos e inaptitudes. Naturalmente, no siempre los guslos corresponden a las aptiludes. y. tratándose de adolescentes. ir contra sus inclinaciones es a menudo deprimirles o quilarles la alegria del trabajo. 
También hay que distinguir sus guslos, sus aspiraciones pro-

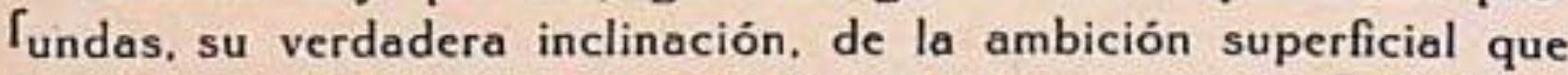
puede resullar de alguna sugestión del medio o de la lectura.

Este interrogatorio del sujeto se complementará con el del medio que lo rodea. A este respecto. hay que reconocer que la Escuela tal como está hoy. se halla mal colocada para informar respecto de las amptitudes de sus alumnos, utilizables profesionalmente. Es demasiado libresca, demasiado verbal, no es lo bastante activa y eslá demasiado alejada de la vida. Pero una vez que haya alcanzado a colocarse dentro del margen que las nuevas orientaciones le prescriben. tal como es ya en algunos establecimientos innovadores. la escuela estará mejor situada que nadie para juzgar de las aptitudes, para explorarlas y aún estimularlas.

En la apreciación del individuo por simple observación, puede incurrirse en errores: sin embargo, es el solo medio de conocer lo relativo a su carácter, a su aspecto y a su temperamento.

Méfodo de los tests. - Vamos a ocuparnos del método a cuyo perfeccionamiento tienden todos los esfuerzos, el mélodo mediante el cual se puede, previo un rápido examen, diagnosticar las aptitudes motrices y mentales de un individuo.

Tomamos la palabra aptifud en el sentido de toda disposición para ejecular un determinado acto o verificar cierto trabajo. La aptitud es todo carácler psíquico o fisico considerado desde el punto de vista del rendimiento. Hay, pues:

Aptitudes sensibles,

Aptiludes motrices.

Aptitudes intelectuales $y$

Aptitudes afectivas.

El problema que se presenta es el de eslablecer tests capaces de evidenciar y medir estas aptitudes. El método de los tests tiene la ventaja de ser:

1. Objefivo: sus resultados son independientes de la apreciación del sujeto o del experimentador: 
2. Determina directamente, en un tiempo relativamente corto, lo que es capaz de hacer el individuo;

3. Permite tomar una medida de la capacidad psicológica esludiada:

4. Permile, si las necesidades se basan en una unidad común, establecer comparaciones entre los resultados oblenidos por investigadores distinlos.

Veamos ahora las diversas categorias de tesís.

Tests profesionales y tests psicológicos,-a) Los tesis profesionales son pruebas elaboradas tomando como punto de partida la estructura o el mecanismo de la profesión, mientras que los psicológicos tienen su punto de partida en la estructura o mecanismo del espiritu.

La mayoria de los tests profesionales tienen por objeto reproducir más o menos exactamente las mismas operaciones que inlegran la profesión, ya sea reproduciendo en toda su compleja realidad y usando las mismas lécnicas, el acto tal como debe ser ejeculado en la futura profesión (tests sintéticos-brutos y iests sintéticos-análogos). o ya sea descomponiendo lo profesión en sus operaciones elementales (fests analiticos).

Asi, al estudiar el problema de las telefonistas, se ha descubierto que necesitan: memoria de cilras. memoria de nombres, atención y rapidez de movimiento. etc.

No olvidemos que antes de servirse de un tests es necesario estar seguro de que éste se dirige hacia esta aptilud, que la solicita, que la moviliza; y. para adquirir esa cerleza, debe ponérselos a prueba para descubrir su exacla significación. testar los tests.

b) Los fests psicológicos son pruebas destinadas a medir las capacidades psicológicas simples (agudeza audiliva, efc.) o más - menos complejas (aptitud para el cálculo, el dibujo. elc.)

Desde el punto de vista de la orientación profesional, se pueden subdividir en 
Tests de inteligencia general $\left\{\begin{array}{c}\text { Tests de inteligencia global. } \\ \text { Tests de inteligencia integral ge- } \\ \text { neral. }\end{array}\right.$ Tesis de aptitudes especiales $\left\{\begin{array}{l}\text { Tesis de conocimientos. } \\ \text { Tests: de aptitudes naturales. }\end{array}\right.$

Los tests de inteligencia general son los destinados a apreciar el grado de inteligencia. Si se trata, por ejemplo, de un niño. se nos dirá si está retrasado o adelantado y de cuántós años, o bien, cuál es el grado de desarrollo de su inteligencia.

Los tests de aplitudes tienden, por el contrario. a determinar qué clase de inteligencia es la que posee el sujeto (si es o no artista, si tiene o no capacidad para las matemáticas, si posee memoria, elc.)

a) La inteligencia general se determina, a partir de los trabajos de Binet y Simon, haciendo ejecutar al sujeto pruebas que no son de inteligencia pura, como por ejemplo: ordenar pesos, copiar dibujos, etc.

Al calcular el promedio de los resultados obtenidos mediante estas diversas pruebas. se determina una cilra que expresa la inteligencia general del sujelo. Esto nos prueba que en toda operación mental interviene un poco la inteligencia.

Pero no sólo basta reconocer el grado de inteligencia general; hay que determinar también la calidad de esta inteligencia general. pues problemas idénticos en su estructura lógica, son resueltos de distinto modo por individuos diferentes, según la naturaleza del objeto del problema.

Así, una persona tendrá más aptitud para los problemas abstractos, otra para los concrelos, unos para las matemáticas y otros para los problemas literarios.

b) Según que las aptitudes sean naturales o adquiridas. tenemos dos clases de tests de aplitudes: $1 .^{\circ}$, Tests de conocimientos: $2^{\circ}$. Tests de aptitudes naturales. 
1. Los tests de conocimientos pueden referirse a los conocimientos adquiridos espontáneamente por el contacto con el medio ambiente, - o a los conocimientos adquiridos en la escuela.

2. Los tests de aptitudes naturales determinan las aptitudes psicológicas y las capacidades propias del individuo. (El llamar naturales a estas aptitudes no quiere decir que dependen únicamente de la herencia y que no sean en nada consecuencia de la educación, porque un individuo es a la vez función de la herencia y del medio).

En cuanto a la determinación del carácter, a la medición de la personalidad en conjunto. se han confeccionado en estos últimos años pruebas o tests que todavia son considerados solamente como ensayos.

Sobre la manera de hacer los tests: Es ésta una tarea sumamente complicada. Veamos sólo algunos trazos generales.

En primer lugar, es necesario que el individuo sometido a la prueba la ejecute con buena voluntad y se encuentre en un estado snormals, en cuanto sea posible.

Ejecutada la prueba. es decir, hecho el tests, hay que proceder a diagnosticar el grado de aptifud de ese individuo, pero en relación con los demás individuos. Por ejemplo: se quiere examinar la memoria de un adolescente. Se le somete a la prueba de repelir las 15 palabras que acaba de oir. El niño repite 9.

Este dato no tendría valor si no se supiera la cantidad media de palabras que son capaces de retener los demás muchachos de su edad, es decir. si está sobre o debajo del promedio.

Esta clasificación se hace generalmente entre cien individuos. A cada una de esas cien divisiones se da el nombre de percentil. Por consiguiente. para diagnosticar un aptitud, hay que buscar el percentil correspondiente al resultado obtenido por el tesits.

\section{Ejemplo:}




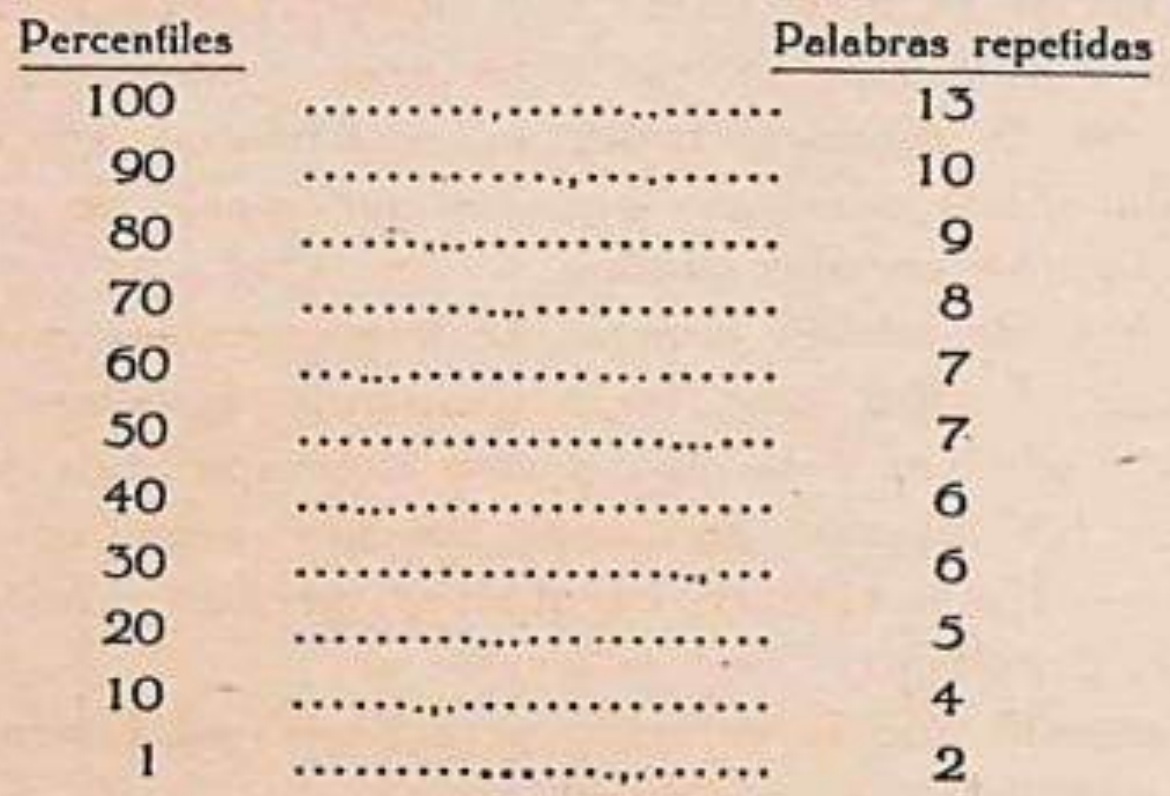

En el caso de la prueba de memoria del niño que repitió 9 palabras, vemos que el grado de su aptitud para la retención inmediata de palabras corresponde a la cifra 80 .

También pueden compararse entre si dos aptitudes de un mismo individuo para ver cuál es la prodominante.

Por ejemplo, un niño que en el test de rapidez de escritura hace 120 letras por minulo y su rapidez en el cálculo es de 4 adiciones por minulo. encontraremos para estos valores los siguientes percentiles:

Memoria de palabras.................. 80

Rapidez de escritura.................. 75

Adiciones ............................. 20

Lo que quiere decir que su aptitud dominante es la memoria, mientras que en el cálculo queda por debajo del promedio.

Estas tablas de percentilaje tienen por objeto dar a los términos muy bueno, bueno. insuficiente, etc., valores exactos que permitan la comparación de psicogramas de una a otra oficina, de uno a otro país.

Respecto al delicado problema de la educabilidad, los experimentos que se han llevado a cabo demuestran que aquellos cuya 
aptitud inicial es más débil, son los que aprovechan más del ejercicio. Naturalmente, esta regla tiene numerosas excepciones. En general, la memoria bruta, la sensibilidad, son muy poco o nada educables: mientras que las funciones de coordinación, motriz, lo son en alto grado.

Es, pues, de interés común, de interés social, que cada hombre ocupe el sitio que le corresponde. Igualmente es conveniente y cada vez está más dentro del espíritu de las democracias, que las diversas funciones sociales sean confiadas a los individuos leniéndose en cuenta sus méritos y no en virtud de cualquier privilegio.

Unicamente una orientación profesional sólidamente elaborada puede acercarnos a este ideal.

JUSTINA OLATE

Alumna del Curso de Francts de la

Escuela de Pedagogia de Concepción. 